\section{Adolescents and young adults with 22 qII deletion syndrome: psychopathology in an at-risk group}

\author{
KATE D. BAKER and DAVID H. SKUSE
}

Microdeletions at chromosome 22q11.2 (22q11 deletion syndrome, velo-cardiofacial syndrome, DiGeorge syndrome; prevalence $\sim 1$ in 4000 ) can cause heart and palate malformations, mild to moderate learning disability and communication impairments (Scambler, 2000). Schizophrenia is a late manifestation in around $30 \%$ of cases, on a par with the risk to offspring of two parents with schizophrenia (Murphy et al, 1999). People with schizophrenia have an 80 -fold increased prevalence of the microdeletion relative to the general population (Karayiorgou et al, 1995). Individuals with schizophrenia and a 22q11.2 microdeletion do not differ from idiopathic cases in age of onset, symptom profiles or medication responses (Bassett et al, 2003). Linkage and association findings also are suggestive of schizophrenia susceptibility loci at 22q (Harrison \& Owen, 2003). This study tested the hypothesis that 22q11 deletion syndrome is associated with specific developmental psychopathology similar to that seen in idiopathic schizophrenia, owing to shared genetic and pathophysiological mechanisms.

\section{METHOD}

\section{Study participants}

This study received ethical approval from the Great Ormond Street Hospital National Health Service Trust research ethics committee. Written informed consent was obtained from parents/carers and from participants aged over 16 years. Individuals with 22q11 deletion syndrome were recruited with the assistance of the UK 22q11 Group (a nationwide family support and information group) and a multidisciplinary clinic. Young people with low average general ability or mild/moderate learning disability but no known neurological condition, genetic syndrome or major sensory impairment were invited to be comparison participants. Sources of these volunteers were mainstream schools $(64 \%)$, colleges of further education $(12 \%)$ and organisations providing leisure services for young people with learning disabilities $(24 \%)$, paralleling the range of current placements of people with $22 \mathrm{q} 11$ deletion syndrome. Twenty-five individuals were recruited for each group. The two groups were well-matched in terms of gender, age (range from 13 to 25 years) and estimated IQ (Table 1).

Given the small sample sizes (although similar to previous studies of this rare condition), it is particularly important for study volunteers in both index and control groups to be representative of their populations. To avoid ascertainment bias towards high rates of disorder in subjects with 22q11 deletion syndrome we publicised the study via a national family support organisation and to all attendees at a multi-disciplinary clinic over several years. For the same reason, we did not recruit participants for either group from clinical services where referral was for behavioural or learning difficulties. Psychiatric disorder and behavioural problems were not exclusion criteria for comparison participants because this would have biased the results towards a low rate of psychopathological disruption, unrepresentative of adolescents and young adults with learning disabilities. To counterbalance self-selection biases, literature about the study provided to potential cases and controls stated that it

Conclusions 22qll deletion syndrome is associated with significant psychopathology prior to adulthood. Double the proportion expected to develop schizophrenia reported psychotic phenomena. These findings imply a continuum of developmental disruption in 22ql I deletion syndrome, associated with declining mental health in early adulthood.

Declaration of interest None.

Table I Study populations

\begin{tabular}{lccc}
\hline & \multicolumn{2}{c}{ Group } & \multirow{2}{*}{ tor $\chi^{21}$} \\
\cline { 2 - 3 } & \multicolumn{2}{c}{ 22qII deletion syndrome $(n=25)$} & Controls $(n=25)$ \\
\hline Gender (male: female) & $15: 10$ & $17: 8$ & $0.35^{\prime}$ \\
Age in years: mean (s.d.) & $16.4(2.0)$ & $16.0(2.9)$ & $1.2^{1}$ \\
Estimated IQ: mean (s.d.) & $66(15)$ & $74(16)$ & $1.9^{1}$ \\
\hline
\end{tabular}

I. All $P>0.05$. 
was important for young people both with and without emotional and behavioural difficulties to take part in the research.

\section{Instruments}

A semi-structured interview of general psychopathology - the Child and Adolescent Psychiatric Assessment (CAPA; Angold et al, 1995) - was selected as the primary instrument for obtaining a descriptive record of each participant's current (previous 3 months) mental state. Interviews were conducted by a research medical student (K.B.) following formal training. They were reviewed by a senior clinician (D.S.) who was masked to the participants' group membership. In all cases, the participant and one or two parents/carers were interviewed using the appropriate CAPA schedule. Interviews took place at the participants' homes, except for five participants who were assessed at Great Ormond Street Hospital. Whenever possible, parents and offspring were interviewed separately. Audio tape recordings and written notes were made during the interview for later coding and scoring. Information obtained via the CAPA was used to derive DSM-IV (American Psychiatric Association, 1994) diagnoses and dimensional symptom scores. A symptom was counted as present for dimensional scoring if the participant met intensity criteria, regardless of whether frequency or duration criteria were met for inclusion of the symptom into a diagnostic algorithm. Estimation of full-scale IQ was carried out using abbreviated forms of age-appropriate Wechsler tests according to published protocols described by Crawford et al (1992) for individuals aged 16 years and over, or by Kaufman et al (1996) for individuals aged under 16 years.

\section{Rating scales}

Premorbid adjustment refers to poor or deteriorating social and adaptive functioning prior to the onset of psychosis. These aspects of the pre-psychotic state may represent a developmental 'negative' syndrome, continuous with that experienced in schizophrenia following onset of psychosis, as shown by longitudinal analyses of psychiatric case notes (Fenton \& McGlashan, 1994). In this study, premorbid adjustment ratings were made using information obtained during the introductory section of the CAPA interview according to modified Cannon-Spoor scales (Cannon et al, 1997).

Schizotypy refers to key personality features and subclinical psychopathological traits that are qualitatively similar to symptoms of a psychotic illness and may predict relative risk of schizophrenia-spectrum illness. They aggregate within families of individuals with schizophrenia and cooccur with neurocognitive dysfunctions (Nuechterlein et al, 2002). In this study, dimensional schizotypy ratings were derived from CAPA interviews according to
ICD-10 (World Health Organization, 1992) criteria for schizotypal disorder (recommended for use in studies of at-risk populations; Gottesman \& ErlenmeyerKimling, 2001). The validity of interviewbased ratings was confirmed by administering a questionnaire measure of schizotypal features (the Junior Schizotypy Schedule; Diduca \& Joseph, 1999) to participants with 22q11 deletion syndrome and controls with adequate literacy levels.

\section{RESULTS}

\section{DSM-IV diagnoses}

\section{and dimensional symptom scores}

Participants with 22q11 deletion syndrome displayed higher rates of psychiatric disorder than individuals of equivalent age and intellectual development: $79 \%$ of $22 \mathrm{q} 11$ deletion syndrome and $21 \%$ of comparison participants fulfilled the criteria for one or more diagnoses (relative risk 14.4; $95 \%$ CI 3.5-58.2). This prevalence in comparison participants is broadly in line with other studies of psychopathology in children and adolescents with learning disability selected from the general population (Emerson, 2003). Table 2 displays the number of specific diagnoses assigned to members of the 22q11 deletion syndrome and control groups and the average symptom counts for each dimension investigated. Odds ratios for the risk of obtaining a diagnosis, given membership of the $22 \mathrm{q} 11$

Table 2 DSM-IV diagnoses and dimensional symptom counts

\begin{tabular}{|c|c|c|c|c|c|c|}
\hline \multirow[t]{2}{*}{ Disorder } & \multicolumn{2}{|c|}{ Number of diagnoses } & \multirow[t]{2}{*}{$\chi^{2}$} & \multicolumn{2}{|c|}{ Mean dimensional symptom count (s.d.) } & \multirow[t]{2}{*}{ Mann-Whitney $U$} \\
\hline & 22qlI deletion syndrome & Controls & & $22 q \mathrm{ll}$ deletion syndrome & Controls & \\
\hline ADHD, inattentive subtype & 12 & 4 & $6.8^{*}$ & $6.6(3.1)$ & $3.4(3.2)$ & $3.1 * *$ \\
\hline Anxiety disorder & 10 & 2 & $6.7^{*}$ & $1.2(2.0)$ & $0.2(0.9)$ & $2.8^{*}$ \\
\hline Separation anxiety & 0 & 0 & & & & \\
\hline Social anxiety & 0 & I & & & & \\
\hline Overanxious & 8 & I & & & & \\
\hline Phobia & 2 & 0 & & & & \\
\hline Mood disorder & 9 & 0 & $\mid I . I^{* *}$ & $2.7(2.4)$ & $0.3(0.9)$ & $4.4^{* *}$ \\
\hline Dysthymia & 4 & 0 & & & & \\
\hline Major depression & 3 & 0 & & & & \\
\hline Hypomania & 2 & 0 & & & & \\
\hline Manic episode & 0 & 0 & & & & \\
\hline Obsessive-compulsive disorder & 2 & 0 & 2.0 & $0.7(\mathrm{I} .5)$ & $0.1(0.4)$ & $2.0^{*}$ \\
\hline Conduct disorder & 0 & 0 & 0 & $0.1(0.4)$ & $0.3(0.6)$ & 1.4 \\
\hline
\end{tabular}

ADHD, attention-deficit/hyperactivity disorder.

$* P<0.05 ; * * P<0.01$. 
deletion syndrome group, were similar for all diagnostic categories: 2.6 for any mood disorder (95\% CI 1.7-3.9), 2.1 for obsessive-compulsive disorder $(95 \%$ CI $1.5-$ 2.8), 2.2 for attention-deficit/hyperactivity disorder, inattentive subtype $195 \%$ CI 1.2-3.9) and 2.1 for any anxiety disorder (95\% CI 1.3-3.4). Comorbidity did not account for these similar risk rates. No participant met the criteria for diagnosis of a psychotic illness.

The 22q11 deletion syndrome group displayed higher symptom scores on all dimensions assessed, except for oppositional defiance/conduct problems. A CAPA symptom summary score, for use in correlational analyses, was calculated for each participant as the sum of $Z$ scores for each DSM-IV symptom dimension (attention, mood, anxiety, obsessive compulsions, oppositional defiance). Mean CAPA symptom summary scores were 1.4 (s.d.=2.9) for the 22q11 deletion syndrome group and -1.3 for the control group (s.d.=1.9). The larger standard deviation in this summary score for the 22q11 deletion syndrome group reflects the considerable variation in severity of psychiatric disorder within this population.

Two specific symptom clusters were reported commonly by participants with 22q11 deletion syndrome. Rapid emotional lability, characterised by frequent, angry outbursts, was a common and intrusive symptom present in 9 out of 25 participants with $22 \mathrm{q} 11$ deletion syndrome (36\%); 12 out of 25 participants with the syndrome (48\%) reported psychosis-like phenomena (corresponding to specific definitions within the CAPA glossary). These were generally of short duration and not perceived as being highly distressing or intrusive. Phenomena were diverse. Examples of delusional thinking included ungrounded fears of being targeted by the police and being watched through closed-circuit television. Examples of perceptual disturbances included auditory (voices talking about the individual, although not necessarily in derogatory terms) and visual hallucinations, in two cases associated with religious imagery and superstitious beliefs not shared by family members. Other abnormal experiences included passivity phenomena, depersonalisation and thought broadcast.

\section{Schizotypy}

A total of 21 out of 25 participants from the $22 \mathrm{q} 11$ deletion syndrome group received at least one positive rating on the schizotypy scale, whereas only 3 out of 25 control participants received any positive ratings. Mean schizotypy scores (out of 9) were 2.8 for 22q11 deletion syndrome participants (s.d.=2.0) and 0.2 for control subjects (s.d. $=0.5$ ) (data not normally distributed, Mann-Whitney $U=59, P<0.0001$ ). To assess the validity of these schizotypy scores derived from CAPA items, correlations between scores on the Junior Schizotypy Schedule questionnaire and on the interview-derived scale were examined. Correlations between scores, for the $22 \mathrm{q} 11$ deletion syndrome and comparison populations combined (Spearman's $\rho=0.34, P=0.052$ ) and the 22q11 deletion syndrome group alone (Spearman's $\rho=0.55, P=0.004)$, confirmed the validity of interview-derived schizotypy ratings.

\section{Premorbid adjustment}

Social-occupational functioning was found to be markedly impaired in participants with $22 \mathrm{q} 11$ deletion syndrome relative to comparison participants (displayed in Table 3), with higher scores on all premorbid adjustment sub-scales except scholastic adaptation. The majority of participants with the syndrome reported impoverished

Table 3 Premorbid adjustment

\begin{tabular}{lccc}
\hline Premorbid adjustment scales & \multicolumn{2}{c}{ Group mean (s.d.) } & \multirow{2}{*}{ Mann-Whitney U } \\
\cline { 2 - 3 } & 22qII deletion syndrome & Controls & \\
\hline Total & $I 1.9(3.7)$ & $7.3(2.2)$ & \multirow{2}{*}{$4.4^{* *}$} \\
Sociability/isolation & $3.5(1.2)$ & $1.6(1.1)$ & $4.5^{* *}$ \\
Peer relations & $3.5(1.4)$ & $1.9(0.8)$ & $4.1^{* *}$ \\
Adaptation to school & $1.8(1.1)$ & $1.9(1.3)$ & 0.4 \\
Interests & $3.2(1.4)$ & $1.9(0.9)$ & $3.4^{* *}$ \\
\hline
\end{tabular}

$* * P<0.01$.

social interactions and peer relationships, associated with lack of interest in or tolerance of others, perceived lack of skill in social situations or rejection by peers. Relative to comparison participants, they also participated far less frequently in organised social activities, such as attending youth clubs or taking part in hobbies. They usually preferred solitary activities, which in some individuals were pursued in an obsessive manner.

\section{Relationships between psychopathology, IQ and age}

There was no significant association between general cognitive impairment and the presence or severity of symptoms and functional impairments. The CAPA symptom summary, the schizotypy score and the total premorbid adjustment score were not correlated with estimated fullscale IQ within the $22 \mathrm{q} 11$ deletion syndrome group. Total premorbid adjustment score was associated with age, with greater impairment seen in older participants with the syndrome (Spearman's $\rho=0.41$, $P=0.046)$. However, there was no significant relationship between age and either CAPA summary score (Spearman's $\rho=0.26, \quad P=0.22$ ) or schizotypy rating (Spearman's $\rho=0.12, P=0.50$ ).

\section{Relationships between psychopathological indices}

Partial correlations were computed for the three summary measures of psychopathology, controlling for age because of the association with premorbid adjustment scores in the 22q11 deletion syndrome group. This analysis was justified only for the $22 \mathrm{q} 11 \mathrm{de}-$ letion syndrome group, because of the limited variance of scores in the comparison group. This indicated a highly significant relationship between schizotypy scores and premorbid adjustment (Spearman's $\rho=0.70, P=0.001$ ) but only a weak trend towards a relationship between CAPA summary score and premorbid adjustment (Spearman's $\rho=0.35, P=0.14$ ). Schizotypy scores and CAPA summary scores also were unrelated to each other (Spearman's $\rho=0.12, P=0.6$ ).

\section{DISCUSSION}

\section{Summary of findings}

This study aimed to characterise psychopathology in adolescents and young adults 
with 22q11 deletion syndrome, and to compare symptom profiles with the developmental trajectory observed in idiopathic schizophrenia. Participants with 22q11 deletion syndrome displayed diverse psychiatric symptoms, the most prevalent of which were mood disruptions, attention deficits and psychotic phenomena. Social and occupational functioning, as assessed by premorbid adjustment scales, was significantly impaired in the 22q11 deletion syndrome group. Equivalent levels of psychopathology and functional difficulty were not seen in comparison participants, and certain symptoms (psychotic experiences and frequent, angry outbursts) were not reported by any control participant in this study. Within the $22 \mathrm{q} 11$ deletion syndrome group, there was no relationship between psychopathology and IQ. Taken together, these findings suggest a specific vulnerability to psychiatric disturbance in $22 \mathrm{q} 11$ deletion syndrome that is independent of learning disability.

The majority of the subjects with $22 \mathrm{q} 11$ deletion syndrome reported symptoms of a schizotypal disorder, unlike matched comparison participants. The severity of their schizotypal features correlated with poorer social functioning and fewer interests. The premorbid adjustment scores (but not CAPA symptom counts or schizotypy) increased with age in the participants with the syndrome, implying greater functional impairment as the individuals approach the time of higher risk for onset of schizophrenia.

For all dimensional symptom and rating scales there was a wider distribution of scores for the 22q11 deletion syndrome group than for controls, but no evidence of bimodal distributions. In the population with the syndrome there were proportionately more young people who describe psychotic symptoms than the $25-30 \%$ who are predicted to go on to develop a psychotic illness. This suggests that there is a continuum of psychotic disorder within the $22 \mathrm{q} 11$ deletion syndrome group from mild to severe, with no clear division between 'affected' and 'unaffected' categories, at least during adolescence.

\section{Comparison with previous studies of 22qll deletion syndrome}

There have been previous reports of moodrelated psychopathology in children and adolescents with 22q11 deletion syndrome (Papolos et al, 1996; Arnold et al, 2001).
We have observed that other disruptions to thought and behaviour also are common. The current study is the first to include a control group of adolescents with learning disability, confirming that these features are not non-specific consequences of neurodevelopmental disorder. Previous casecontrol studies involving mostly younger children with 22q11 deletion syndrome have found no marked differences between groups in rates of psychiatric disorder (Swillen et al, 2001; Feinstein et al, 2002). The contrast with our findings in adolescents could have a variety of explanations. First, critical developmental processes relevant to the emergence of later psychotic illness may occur during later childhood or around the time of puberty. Second, subjective schizotypal phenomena may be present at an earlier age, but individuals are not able to articulate these symptoms until they have reached sufficient levels of cognitive and linguistic attainment. In middle childhood, liability to psychotic symptoms may manifest as non-specific childhood behavioural disturbance, such as separation anxiety, sleep disturbance and somatic complaints (Miller et al, 2002a). We found self-report by adolescents to be an essential resource for determining the presence and content of psychotic phenomena. Previous investigators interviewed parents of individuals with $22 \mathrm{q} 11$ deletion syndrome but not the children themselves, and therefore would not have been made aware of these features.

\section{Comparison with studies of idiopathic schizophrenia}

Schizophrenia is a late psychiatric manifestation of an abnormal neurodevelopmental process, leading to aberrant neurological and psychological functioning at several points along a developmental trajectory. Accordingly, evidence of developmental disruption, in the form of specific cognitive performance decrements and psychiatric symptoms during childhood and adolescence, is detectable prior to the onset of major psychotic illness. Evidence of this type has been obtained by retrospective report (e.g. Cannon et al, 1997) and prospective epidemiological cohort studies (e.g. Done et al, 1994).

The presence of psychotic experiences and other schizotypal phenomena in people with 22q11 deletion syndrome parallels developmental findings in idiopathic schizophrenia. For example, the Dunedin prospective cohort study found an association between self-reported psychotic symptoms at age 11 years and schizophreniform illness (but not mania or depression) at age 26 years (odds ratio 16.4). Nearly half $(42 \%)$ of adult patients reported symptoms at this early age (Poulton et al, 2000). In genetic high-risk studies, the presence of psychosis-like phenomena during childhood and adolescence predicts the likelihood of adult-onset illness (Miller et al, 2002b; Ott et al, 2002). However, the predictive power of these phenomena is not high and there are significant falsepositive and false-negative rates. Psychotic experiences and schizotypal characteristics during adolescence may be relatively common, reflecting neurodevelopmental deviations from the norm that do not necessarily lead to schizophrenia. Conversely, not all adults with schizophrenia report such experiences during adolescence. Therefore, early symptoms are likely to reflect genetically mediated neurodevelopmental disruption, which interacts in a currently unpredictable fashion with later neurobiological and psychosocial events to precipitate psychotic illness in a subset of individuals at high risk.

Our findings suggest that, whereas psychiatric symptoms may occur at at any time during adolescence in 22q11 deletion syndrome, the social and functional impairment associated with them increases over time. Within the limits of a cross-sectional study, it is arguable that the observed association between older age and poorer functional adjustment reflects a process of intra-individual functional decline. Genetic high-risk studies have not as yet collected sufficiently fine-grained data in an adequate sample to observe such a decline in individuals who develop adult-onset psychosis. However, recent clinical research aiming to facilitate early identification and preventive intervention for schizophrenia has identified high-risk groups of young people presenting to psychiatric services with prodromal symptoms. Recent reports from the largest such cohort indicate that, although attenuated psychotic symptoms are moderately predictive of risk of illness within a 12-month period, detection rates are greatly increased when measures of functional capacity and, crucially, recent functional decline are taken into account (Yung et al, 2004).

Longitudinal studies in high-risk groups (both genetically and clinically defined) have shown that psychopathological decline, 
which presages illness-onset, may be mirroring a process of neuropsychological (IQ and memory) and anatomical (medial temporal lobe) deterioration in already at-risk individuals (Cosway et al, 2000; Pantelis et al, 2003). Longitudinal follow-up of the present 22q11 deletion syndrome cohort will determine whether a similar trajectory characterises the emergence of debilitating psychotic illness in syndromal cases. Several small randomised controlled trials have now been carried out within clinically high-risk samples, to determine whether it is possible to prevent or delay deterioration by pharmacological or psychotherapeutic intervention. Findings from these trials have been mixed so far, limited by the small sample size and short periods of follow-up, but ongoing development of detection criteria and treatment protocols may yield results of clinical benefit to people with $22 \mathrm{q} 11$ deletion syndrome.

\section{Implications for genetics, neurodevelopment and schizophrenia}

Specific neurocognitive substrates appear to underlie susceptibility to psychosis. The mechanisms involved in mediating risk for psychiatric illness in people with $22 \mathrm{q} 11$ deletion syndrome are still speculative. Perhaps haploinsufficiency for the protein product of a key gene at the 22q11 locus impairs the development of a critical neurocognitive mechanism that has a wideranging effect on psychological function. Individual symptoms arise stochastically, as a consequence of unpredictable environmental interactions with an abnormal trajectory of emotional-behavioural maturation. This model is supported partially by the present findings: symptoms reported by participants with $22 \mathrm{q} 11$ deletion syndrome varied widely in both type and severity. Alternatively, haploinsufficiency of a key gene in the $22 \mathrm{q} 11$ region may render vulnerable the development of many different neurocognitive processes. Each symptom cluster (e.g. attention-deficit, psychotic phenomena) could have its origin in different impairment processes of maturation or function. This hypothesis is supported by the consistency between participants in the characteristics of certain symptoms, such as angry outbursts and psychosis-like phenomena, that suggest disruption to context-dependent emotion regulation and reality monitoring. Early

\section{CLINICAL IMPLICATIONS}

- Adolescents and young adults with chromosome 22qll deletion syndrome experience diverse psychiatric symptoms, many of which are revealed only by personal interview.

Schizotypal phenomena similar to those observed in association with psychosis risk in the general population affect up to half of adolescents with 22qll deletion syndrome.

- Strategies under development for the early identification of psychosis risk and provision of prophylactic treatments to young people in the general population may be applicable to individuals with 22 qll deletion syndrome.

\section{LIMITATIONS}

- Interpretation of findings is limited by lack of an idiopathic early-onset psychosis group or familial high-risk group with which to make direct comparisons with 22qll deletion syndrome.

- Interviews were conducted by one investigator, who could not be masked to diagnosis or to responses given by additional informants.

- A longitudinal study is required to determine the predictive value of adolescent psychiatric symptoms for the later emergence of schizophrenia-spectrum illness in 22qll deletion syndrome.

KATE D. BAKER, PhD, DAVID H. SKUSE, FRCPsych, Behavioural and Brain Sciences Unit, Institute of Child Health, University College London, London, UK

Correspondence: Kate Baker, Behavioural and Brain Sciences Unit, Institute of Child Health, University College London, 30 Guilford Street, London WCIN IEH, UK. E-mail: k.baker@ich.ucl.ac.uk

(First received 21 June 2004, final revision 7 October 2004, accepted 12 October 2004)

indications are that psychotic phenomena in the adolescent cohort with $22 \mathrm{q} 11$ deletion syndrome are associated with abnormal perceptual processing and impaired working memory systems. We have evidence that distinct impairments in event-related electroencephalogram potentials (mismatch negativity), evoked in response to unpredictable change in an auditory stream, closely resemble abnormalities seen in idiopathic schizophrenia (details available from the authors upon request). The similarity in psychopathological features during adolescence and young adulthood reported here provides additional support for the hypothesis that parallel neurodevelopmental disruption occurs in $22 \mathrm{q} 11$ deletion syndrome and in idiopathic schizophrenia. Further investigation of gene-brain-behaviour relationships in people with $22 \mathrm{q} 11$ deletion syndrome may add much to our understanding of developmen- tal events contributing to the risk of psychotic disorders and the mechanisms by which genetic factors influence individual risk.

\section{REFERENCES}

American Psychiatric Association (1994) Diagnostic and Statistical Manual of Mental Disorders (4th edn) (DSM-IV). Washington, DC: APA.

Angold, A., Prendergast, M., Cox, A., et al (1995)

The Child and Adolescent Psychiatric Assessment (CAPA). Psychological Medicine, 25, 739-753.

Arnold, P. D., Siegel-Bartelt, J., Cytrynbaum, C. et al (200I) Velo-cardio-facial syndrome: implication of microdeletion 22qll for schizophrenia and mood disorders. American Journal of Medical Genetics, 105 354-362.

Bassett, A., Chow, E.W., Abdel Malik, P., et al (2003) The schizophrenia phenotype in 22qll deletion syndrome. American Journal of Psychiatry, 160 1580-1586.

Cannon, M., Jones, P., Gilvarry, C., et al (1997) Premorbid social functioning in schizophrenia and 
bipolar disorder: similarities and differences. American Journal of Psychiatry, 154, 1544-1550.

\section{Cosway, R., Byrne, M., Clafferty, R., et al (2000)} Neuropsychological change in young people at high risk for schizophrenia: results from the first two neuropsychological assessments of the Edinburgh High-Risk Study. Psychological Medicine, 30, IIII-II2I.

Crawford, J. R., Allen, K. M. \& Jack, A. M. (1992) Short-forms of the UK WAIS-R: regression equations and their predictive validity in a general population sample. British Journal of Clinical Psychology, 31, 191-202.

Diduca, D. \& Joseph, S. (1999) Assessing schizotypal traits in 13-18 year olds: revising the JSS. Personality and Individual Differences, 27, 673-682.

Done, D. J., Crow, T. J., Johnstone, E. C., et al (1994) Childhood antecedents of schizophrenia and affective illness: social adjustment at ages 7 and II. BMJ, 309, 699-703.

Emerson, E. (2003) Prevalence of psychiatric disorders in children and adolescents with and without intellectual disability. Journal of Intellectual Disability Research, 47, 5I-58.

Feinstein, C., Eliez, S., Blasey, C., et al (2002) Psychiatric disorders and behavioral problems in children with velocardiofacial syndrome: usefulness as phenotypic indicators of schizophrenia risk. Biological Psychiatry, 5I, 312-318.

Fenton, W. S. \& McGlashan, T. H. (1994) Antecedents, symptom progression, and long-term outcome of the deficit outcome in schizophrenia. American Journal of Psychiatry, I5I, 35I-356.

Gottesman, I. I. \& Erlenmeyer-Kimling, L. (200I) Family and twin strategies as a head start in defining prodromes and endophenotypes for hypothetical earlyinterventions in schizophrenia. Schizophrenia Research 5I, 93-102.

Harrison, P. J. \& Owen, M. J. (2003) Genes for schizophrenia? Recent findings and their pathophysiological implications. Lancet, 361, 417-419.

Karayiorgou, M., Morris, M. A., Morrow, B., et al (1995) Schizophrenia susceptibility associated with interstitial deletions of chromosome 22ql I. Proceedings of the National Academy of Sciences of the USA, 92 7612-7616.

Kaufman, A. S., Kaufman, J. C., Balgopal, R., et al (1996) Comparison of three WISC-III short forms: weighing psychometric, clinical, and practical factors. Journal of Clinical Child Psychology, 25, 97-105.

Miller, P. M., Byrne, M., Hodges, A., et al (2002a) Childhood behaviour, psychotic symptoms and psychosis onset in young people at high risk of schizophrenia: early findings from the Edinburgh High-Risk Study.

Psychological Medicine, 32, 173-179.

Miller, P. M., Lawrie, S. M., Byrne, M., et al (2002b) Self-rated schizotypal cognitions, psychotic symptoms and the onset of schizophrenia in young people at high risk of schizophrenia. Acta Psychiatrica Scandinavica, 105 34I-345.

Murphy, K. C., Jones, L. A. \& Owen, M. J. (1999) High rates of schizophrenia in adults with velo-cardio-facial syndrome. Archives of General Psychiatry, 56, 940-945.

Nuechterlein, K. H., Asarnow, R. F., Subotnik, K. L., et al (2002) The structure of schizotypy: relationships between neurocognitive and personality disorder features in relatives of schizophrenic patients in the UCLA Family Study. Schizophrenia Research, 54, 121-130.
Ott, S. L., Roberts, S., Rock, D., et al (2002) Positive and negative thought disorder and psychopathology in childhood among subjects with adulthood schizophrenia. Schizophrenia Research, 58, 231-239.

Pantelis, C., Velakoulis, D., McGorry, P. D., et al (2003) Neuroanatomical abnormalities before and after onset of psychosis: a cross-sectional and longitudinal MRI comparison. Lancet, 36I, 28I-288.

Papolos, D. F., Faedda, G. L., Veit, S., et al (1996) Bipolar spectrum disorders in patients diagnosed with velo-cardio-facial syndrome: does a hemizygous deletion of chromosome 22qll result in bipolar affective disorder? American Journal of Psychiatry, 153, I54I-1547.

Poulton, R., Caspi, A., Moffitt, T. E., et al (2000) Children's self-reported psychotic symptoms and adult schizophreniform disorder: a 15 year longitudinal study. Archives of General Psychiatry, 57, 1053-1058.

Scambler, P. J. (2000) The 22qll deletion syndromes. Human Molecular Genetics, 9, 2421-2426.

Swillen, A., Devriendt, K., Ghesquiere, P., et a (200I) Children with a 22qll deletion versus children with a speech-language impairment and learning disability: behavior during primary school age. Genetic Counselling, 12, 309-317.

World Health Organization (1992) Tenth Revision of the International Classification of Diseases and Related Health Problems (ICD-10). Geneva: WHO.

Yung, A. R., Phillips, L. J., Yuen, H. P., et al (2004) Risk factors for psychosis in an ultra high-risk group: psychopathology and clinical features. Schizophrenia Research, 67, $131-142$ 